Received: 11.08 .2017

Revised: 19.03.2018

Accepted: 30.03 .2018

DOI: $10.17804 / 2410-9908.2018 .2 .029-041$

\title{
ASYMPTOTIC ANALYSIS OF THE STRESS FIELD AT A CRACK TIP IN A LINEARLY ELASTIC MATERIAL: EXPERIMENTAL DETERMINATION OF WILLIAMS EXPANSION COEFFICIENTS
}

\author{
L. V. Stepanova \\ S. P. Korolev Samara National Research University, \\ 34 Moskovskoe Hw, Samara, 443086, Russian Federation \\ iDhttp://orcid.org/0000-0002-6693-3132 ه $\underline{\text { stepanovalv@samsu.ru }}$ \\ Corresponding author. E-mail: stepanovalv@samsu.ru \\ Address for correspondence: Moskovskoe shosse, 34, 443086, Samara, Russian Federation \\ Tel.: +7927 7522102
}

The paper deals with analytical determination of the coefficients of the complete Williams asymptotic expansion for the stress field at the tips of two collinear cracks in an infinite elastic plate under mixed-mode (Mode I and Mode II) loading. A method for the determination of the coefficients is presented, which is based on the classical complex representation of the Kolosov-Muskhelishvili solution and its series expansion in the vicinity of the crack tip. An analytical representation of the coefficients of the complete Williams asymptotic expansion ( $T$-stresses and higher-order approximation coefficients) as functions of applied loads, crack lengths and inter-crack distances is found for a plate with two collinear cracks. The paper presents experimental results on the photoelastic study of the stress field at the crack tips of two collinear cracks in a plate made of an optically active material (epoxy resin). It is demonstrated that higher-order approximations must be kept in the Williams asymptotic expansion for the accurate description of the stress field and the correct processing of the interference fringe pattern. The longer the distance from the crack tip to the point on the isochromatic fringe, the more terms of the asymptotic expansion need to be kept.

Key words: Williams asymptotic expansion, experimental determination of the coefficients of the Williams power series expansion, photo elasticity method, higher-order terms of the Williams asymptotic expansion.

\section{References}

1. Hello G., Tahar M.-B., Roelandt J.-M. Analytical determination of coefficients in crack-tip stress expansions for a finite crack in an infinite plane medium. International Journal of Solids and Structures, 2012, vol. 49, iss. 3-4, pp. 556-566. DOI: 10.1016/j.ijsolstr.2011.10.024.

2. Stepanova L.V. Matematicheskie metody mekhaniki razrusheniya [Mathematical methods of fracture mechanics]. Samara, Samarskii universitet Publ., 2006, 232 p. (In Russian).

3. Muskhelishvili N.I. Nekotorye osnovnye zadachi matematicheskoy teorii uprugosti [Some basic problems of the mathematical theory of elasticity]. Moscow, Nauka Publ., 1966, 708 p. (In Russian).

4. Gupta M., Alderliesten R.C., Benedictus R. A review of T-stress and its effects in. fracture mechanics. Engineering Fracture Mechanics, 2015, vol. 134, 218-241. DOI: 10.1016/ j.engfracmech.2014.10.013 
5. Matvienko Yu.G. The effect of the non-singular t-stress components on crack tip plastic zone under mode I loading. Procedia Materials Science, 2014, vol. 3, pp. 141-146. DOI: $10.1016 /$ j.mspro.2014.06.026.

6. Stepanova L.V., Adylina E.M. Stress-strain state in the vicinity of a crack tip under mixed loading. Journal of Applied Mechanics and Technical, 2014, vol. 55, iss. 5, pp. 181-194. DOI: $10.1134 / \mathrm{S} 0021894414050186$.

7. Kobayashi A.S. Handbook on Experimental Mechanics, Society for Experimental Mechanics, Seattle, 1987. 
Подана в журнал: 11.08 .2017

УДК 539.3

DOI: $10.17804 / 2410-9908.2018 .2 .029-041$

\title{
АСИМПТОТИЧЕСКИЙ АНАЛИЗ ПОЛЯ НАПРЯЖЕНИЙ У ВЕРШИНЫ ТРЕЩИНЫ В ЛИНЕЙНО УПРУГОМ МАТЕРИАЛЕ: ЭКСПЕРИМЕНТАЛЬНОЕ ОПРЕДЕЛЕНИЕ КОЭФФИЦИЕНТОВ РАЗЛОЖЕНИЯ М. УИЛЬЯМСА
}

\author{
Л. В. Степанова \\ Самарский национальный исследовательский университет имени академика С. П. Королева, \\ 443086, Московское шоссе, 34, Самара, Российская Федерация \\ Dhttp://orcid.org/0000-0002-6693-3132 @ stepanovalv@ samsu.ru \\ Ответственный автор. Электронная почта: stepanovalv@ samsu.ru \\ Адрес для переписки: Московское шоссе, 34, 443086, г. Самара, Российская Федерация \\ Тел.: +7-927-752-21-02
}

Работа посвящена аналитическому определению коэффициентов полного асимптотического разложения М. Уильямса поля напряжений у вершин двух коллинеарных трещин в бесконечной пластине, находящейся в условиях смешанного нагружения. Представлен метод вычисления коэффициентов асимптотического разложения М. Уильямса, базирующийся на классическом комплексном представлении решения Колосова-Мусхелишвили и его разложении в ряд в окрестности вершины трещины. Найдены аналитические зависимости коэффициентов полного асимптотического разложения М. Уильямса ( $T$-напряжений и коэффициентов высших приближений) от приложенной нагрузки, длин трещин и расстояний между ними для пластины с двумя коллинеарными трещинами. В работе представлены результаты экспериментального исследования поля напряжений у вершин двух коллинеарных трещин в пластине из оптически активного материала (эпоксидной смолы) методом фотоупругости. Показано, что для аккуратного описания поля напряжений и правильной обработки интерференционной картины полос в асимптотическом разложении М. Уильямса следует удерживать высшие приближения. Чем дальше точка изохроматической полосы находится от вершины трещины, тем больше слагаемых в асимптотическом разложении необходимо удерживать.

Ключевые слова: асимптотическое разложение М. Уильямса, экспериментальное определение коэффициентов разложение М, Уильямса, метод фотоупругости, высшие приближения разложения М. Уильямса

\section{1. Введение}

Полное асимптотическое представление поля напряжений в окрестности вершины трещины, содержащее высшее приближение, - асимптотическое решение М. Уильямса, является общим описанием поля напряжений вблизи вершины трещины в линейно упругом изотропном материале $[1,2]$. Асимптотическое разложение М. Уильямса для каждой конфигурации образца содержит последовательность коэффициентов, зависящих от геометрических параметров образца и системы приложенных нагрузок. Широко известны аналитические представления для первых двух слагаемых в асимптотических разложениях поля напряжений: для коэффициентов интенсивности напряжений и $T$-напряжений для целого ряда конфигураций образцов с трещинами [1]. Более сложную задачу представляет собой определение коэффициентов высших приближений как функций приложенной нагрузки и геометрии рассматриваемого образца с трещиной. В настоящей статье приводятся аналитические выражения для коэффициентов полного асимптотического разложения М. Уильямса 
поля напряжений в окрестности вершин двух коллинеарных трещин в бесконечной пластине, находящейся в условиях смешанного деформирования, когда на бесконечности приложены растягивающая и сдвиговая нагрузки. Идентификация коэффициентов полного асимптотического разложения М. Уильямса основана на точном аналитическом решении задачи, полученным с помощью классического комплексного представления решения плоской задачи теории упругости [3]. Используя метод разложения по собственным функциям, М. Уильямс представил функцию напряжений Эри в окрестности вершины трещины в форме $\chi(r, \theta)=\sum_{i} f_{i}(\theta) r^{\lambda_{i}}$, где $f_{i}(\theta)$ и $\lambda_{i}$ - собственные функции и собственные значения соответственно. При этом компоненты тензора напряжений в окрестности вершины трещины принимают вид:

$$
\sigma_{i j}(r, \theta)=\sum_{m=1}^{2} \sum_{k=-\infty}^{\infty} a_{k}^{m} f_{k}^{m, i j}(\theta) r^{k / 2-1}
$$

где индекс $m$ отвечает типу нагружения; коэффициенты $a_{k}^{m}$ зависят от геометрии образца с трещиной и от системы приложенных нагрузок; $f_{k}^{m, i j}(\theta)$ - универсальные угловые распределения, определяемые в ходе решения краевой задачи. Хорошо известны угловые распределения напряжений в (1):

$$
\begin{aligned}
& f_{k}^{1,11}(\theta)=k\left[\left(2+k / 2+(-1)^{k}\right) \cos (k / 2-1) \theta-(k / 2-1) \cos (k / 2-3) \theta\right] / 2, \\
& f_{k}^{1,22}(\theta)=k\left[\left(2+k / 2-(-1)^{k}\right) \cos (k / 2-1) \theta+(k / 2-1) \cos (k / 2-3) \theta\right] / 2, \\
& f_{k}^{1,12}(\theta)=k\left[(k / 2-1) \sin (k / 2-3) \theta-\left(k / 2+(-1)^{k}\right) \sin (k / 2-1) \theta\right] / 2, \\
& f_{k}^{2,11}(\theta)=-k\left[\left(2+k / 2-(-1)^{k}\right) \sin (k / 2-1) \theta-(k / 2-1) \sin (k / 2-3) \theta\right] / 2, \\
& f_{k}^{2,22}(\theta)=-k\left[\left(2-k / 2+(-1)^{k}\right) \sin (k / 2-1) \theta+(k / 2-1) \sin (k / 2-3) \theta\right] / 2, \\
& f_{k}^{2,12}(\theta)=k\left[(k / 2-1) \cos (k / 2-3) \theta-\left(k / 2-(-1)^{k}\right) \cos (k / 2-1) \theta\right] / 2 .
\end{aligned}
$$

Характеристики краевой задачи механики трещин, такие как геометрия области с разрезом, природа и интенсивность приложенной нагрузки, не входят ни в радиальное, ни в угловое распределение компонент тензора напряжений. Таким образом, все разнообразие задач механики разрушения учитывается коэффициентами $a_{k}^{m}$. В настоящее время в механике разрушения сложилось ясное понимание необходимости удержания высших слагаемых в полном асимптотическом разложении М. Уильямса (1-3) и, следовательно, о необходимости определения его коэффициентов $a_{k}^{m}$ для различных образцов, наиболее часто используемых в инженерной практике [4-6]. Одним из перспективных методов, позволяющих найти аналитические выражения для коэффициентов асимптотических разложений, является метод, основанный на комплексном представлении Колосова - Мусхелишвили [3], и последующем сращивании аналитического решения, полученного с помощью теории функции комплексного переменного, с асимптотическим решением М. Уильямса. В настоящей работе получены аналитические выражения коэффициентов полного асимптотического разложения М. Уильямса для бесконечной пластины с двумя коллинеарными трещинами (рис. 1). 


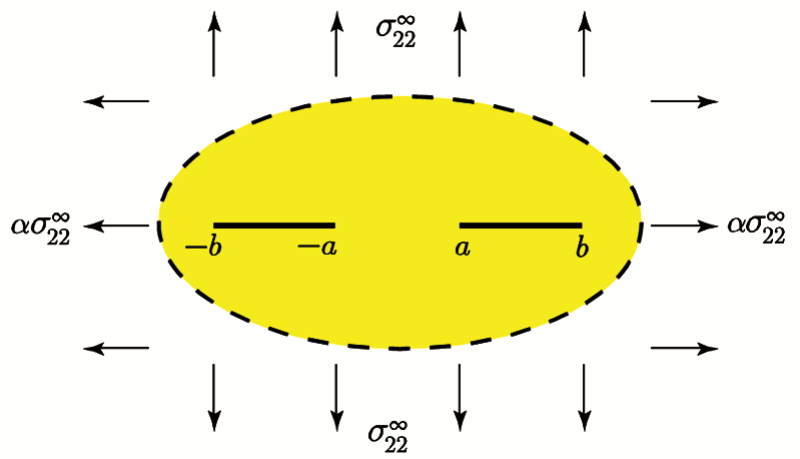

$a$

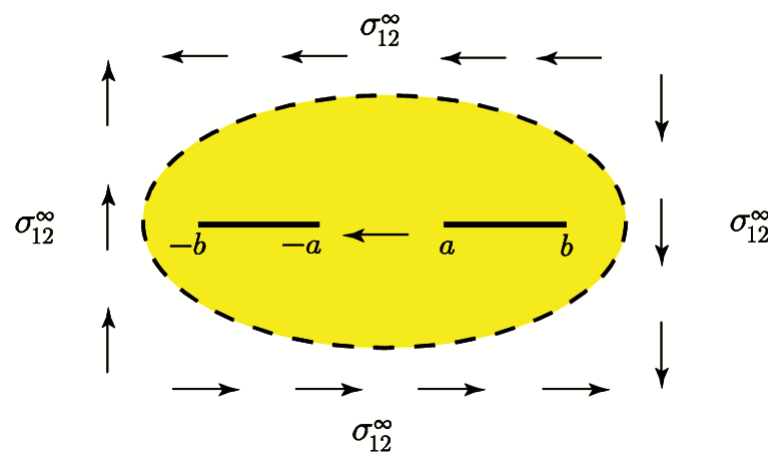

$\sigma$

Рис. 1. Симметричное и антисимметричное нагружение пластины с двумя коллинеарными трещинами

\section{2. Комплексное представление решения для бесконечной пластины с двумя коллинеарными трещинами}

В случае двухосного симметричного растяжения пластины (рис. 1 a) комплексное представление поля напряжений имеет вид $[1,3]$ :

$$
\begin{gathered}
\sigma_{11}^{1}(z)=2 \operatorname{Re}\left[\varphi_{1}^{\prime}(z)\right]-2 x_{2} \operatorname{Im}\left[\varphi_{1}^{\prime \prime}(z)\right]+C_{1}, \sigma_{22}^{1}(z)=2 \operatorname{Re}\left[\varphi_{1}{ }^{\prime}(z)\right]+2 x_{2} \operatorname{Im}\left[\varphi_{1}{ }^{\prime \prime}(z)\right]-C_{1}, \\
\sigma_{12}^{1}(z)=-2 x_{2} \operatorname{Re}\left[\varphi_{1}^{\prime \prime}(z)\right] .
\end{gathered}
$$

Комплексный потенциал $\varphi_{1}^{\prime}(z)$ для пластины с двумя трещинами определяется формулой [4]:

$$
\varphi_{1}^{\prime}(z)=\frac{\sigma_{22}^{\infty}}{2} \frac{z^{2}-c_{2}}{\sqrt{\left(z^{2}-a^{2}\right)\left(z^{2}-b^{2}\right)}}+(\alpha-1) \frac{\sigma_{22}^{\infty}}{4}, C_{1}=(\alpha-1) \sigma_{22}^{\infty} / 2, c_{2}=b^{2} \frac{E(\pi / 2, k)}{F(\pi / 2, k)}, k=\sqrt{1-a^{2} / b^{2}},
$$

где $E(\pi / 2, k), F(\pi / 2, k)$ - полные эллиптические интегралы третьего и первого рода. Выражения (4) для симметричного нагружения трещины (рис. 2 a) принимают вид:

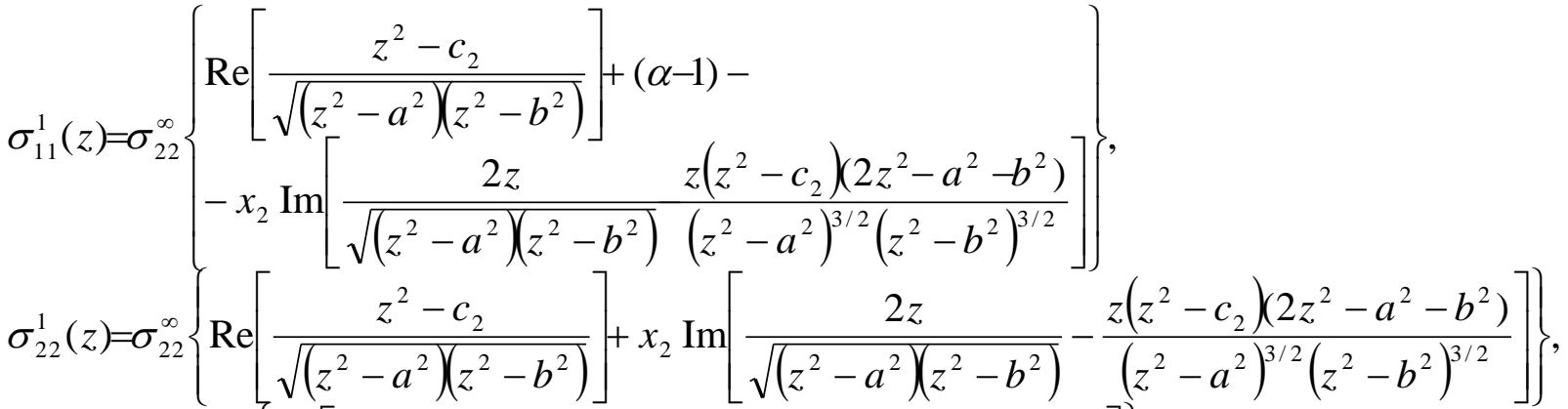

$$
\begin{aligned}
& \sigma_{12}^{1}(z)=-\sigma_{22}^{\infty} x_{2}\left\{\operatorname{Re}\left[\frac{2 z}{\sqrt{\left(z^{2}-a^{2}\right)\left(z^{2}-b^{2}\right)}}-\frac{z\left(z^{2}-c_{2}\right)\left(2 z^{2}-a^{2}-b^{2}\right)}{\left(z^{2}-a^{2}\right)^{3 / 2}\left(z^{2}-b^{2}\right)^{3 / 2}}\right]\right\} \text {. }
\end{aligned}
$$


Для поперечного сдвига (рис. 2 в) поле напряжений определяется комплексным потенциалом:

$$
\begin{aligned}
& \sigma_{11}^{2}(z)=2 \operatorname{Re}\left[\phi_{2}{ }^{\prime}(z)\right]-2 x_{2} \operatorname{Im}\left[\phi_{2}{ }^{\prime \prime}(z)\right], \sigma_{22}^{2}(z)=2 x_{2} \operatorname{Im}\left[\phi_{2}{ }^{\prime \prime}(z)\right], \\
& \sigma_{12}^{2}(z)=-2 \operatorname{Im}\left[\phi_{2}{ }^{\prime}(z)\right]-2 x_{2} \operatorname{Re}\left[\phi_{2}{ }^{\prime \prime}(z)\right]-C_{2} .
\end{aligned}
$$

Комплексный потенциал $\varphi_{2}{ }^{\prime}(z)$ в соответствии с [3] имеет вид:

$$
\varphi_{2}^{\prime}(z)=-i\left(\sigma_{12}^{\infty} / 2\right)\left[\left(z^{2}-c_{2}\right) / \sqrt{\left(z^{2}-a^{2}\right)\left(z^{2}-b^{2}\right)}\right]+i \sigma_{12}^{\infty} / 2, \quad C_{2}=\sigma_{12}^{\infty} .
$$

Компоненты тензора напряжений для антисимметричного нагружения трещины принимают вид:

$$
\begin{aligned}
& \sigma_{11}^{2}(z)=\sigma_{12}^{\infty}\left\{2 \operatorname{Im}\left[\frac{z^{2}-c_{2}}{\sqrt{\left(z^{2}-a^{2}\right)\left(z^{2}-b^{2}\right)}}\right]+x_{2} \operatorname{Re}\left[\frac{2 z}{\sqrt{\left(z^{2}-a^{2}\right)\left(z^{2}-b^{2}\right)}}-\frac{z\left(z^{2}-c_{2}\right)\left(2 z^{2}-a^{2}-b^{2}\right)}{\left(z^{2}-a^{2}\right)^{3 / 2}\left(z^{2}-b^{2}\right)^{3 / 2}}\right]\right\}, \\
& \sigma_{22}^{2}(z)=-\sigma_{12}^{\infty} x_{2} \operatorname{Re}\left[\frac{2 z}{\sqrt{\left(z^{2}-a^{2}\right)\left(z^{2}-b^{2}\right)}}-\frac{z\left(z^{2}-c_{2}\right)\left(2 z^{2}-a^{2}-b^{2}\right)}{\left(z^{2}-a^{2}\right)^{3 / 2}\left(z^{2}-b^{2}\right)^{3 / 2}}\right], \\
& \sigma_{12}^{2}(z)=\sigma_{12}^{\infty}\left\{\operatorname{Re}\left[\frac{z^{2}-c_{2}}{\sqrt{\left(z^{2}-a^{2}\right)\left(z^{2}-b^{2}\right)}}\right]-x_{2} \operatorname{Im}\left[\frac{2 z}{\sqrt{\left(z^{2}-a^{2}\right)\left(z^{2}-b^{2}\right)}}-\frac{z\left(z^{2}-c_{2}\right)\left(2 z^{2}-a^{2}-b^{2}\right)}{\left(z^{2}-a^{2}\right)^{3 / 2}\left(z^{2}-b^{2}\right)^{3 / 2}}\right]\right\} .
\end{aligned}
$$

Сравнивая асимптотическое решение М. Уильямса с угловыми распределениями (1) и (5), можно найти $K_{I}(b)=\sqrt{\pi} \sigma_{22}^{\infty} b^{3 / 2}[1-E(\pi / 2, k) / F(\pi / 2, k)] / \sqrt{b^{2}-a^{2}}, T=(\alpha-1) \sigma_{22}^{\infty}$.

\section{3. Экспериментальное исследование пластины с двумя коллинеарными трещинами (метод цифровой фотоупругости)}

К числу актуальных направлений исследований в механике разрушения относится многопараметрический анализ поля напряжений в окрестности вершины трещины или углового выреза. Поле напряжений вблизи вершины трещины можно описать с помощью полного асимптотического разложения М. Уильямса $[1,2]$. Проблему составляет определение коэффициентов асимптотических разложений (коэффициентов интенсивности напряжений, $T$-напряжений и коэффициентов высших приближений) как функций приложенной нагрузки и геометрических параметров образца с трещиной. В настоящей работе для вычисления коэффициентов полных асимптотических разложений М. Уильямса был использован метод фотоупругости [3-5], в рамках которого можно получить распределение изохроматических полос в окрестности вершины трещины. Для проверки результатов обработки экспериментальных данных была проведена серия конечно-элементных расчетов для серии экспериментальных образцов с трещинами и надрезами. Необходимо отметить, что метод фотоупругости и оптико-механический закон, дающий математическую основу определения коэффициентов асимптотических разложений компонент тензора напряжений и вектора перемещений, широко используется в настоящее время в механике деформируемого твердого тела. К преимуществам метода фотоупругости относятся: 1) возможность формулировки оптикомеханического закона для любого наперед заданного количества экспериментальных точек; 2) тщательный анализ функции формы, фигурирующей в выражениях для коэффициентов интенсивности напряжений (в настоящее время предложены новые экспериментальные об- 
разцы, отличающиеся постоянным значением функции формы, например, надрезанный полудиск для испытания на сжатие); 3) возможность быстрой обработки экспериментальной информации с помощью пакетов прикладных программ.

Настоящая работа представляет собой попытку вычисления коэффициентов в высших приближениях полного асимптотического разложения М. Уильямса (1), (2) на основе методов фотоупругости и метода конечного элемента с помощью алгоритма, специально разработанного для вычисления данных коэффициентов.

Основываясь на классической концепции фотоупругости, положение изохроматической полосы определяется с помощью оптико-механического закона Вертгейма (таким образом, интерференционная картина дает возможность получить линии равных разностей главных напряжений в модели):

$$
\left(\frac{N f_{\sigma}}{h}\right)^{2}=\left(\sigma_{11}-\sigma_{22}\right)^{2}+4 \sigma_{12}^{2},
$$

где $N$ представляет собой порядок изохроматической полосы; $f_{\sigma}-$ оптическая постоянная материала, характеризуемая разностью главных напряжений, необходимой для изменения порядка полос на единицу в модели единичной толщины. Описываемый ниже метод базируется на функции:

$$
g_{m}=\left(\frac{\sigma_{11}-\sigma_{22}}{2}\right)_{m}^{2}+\left(\sigma_{12}\right)_{m}^{2}-\left(\frac{N_{m} f_{\sigma}}{2 h}\right)^{2} .
$$

$a_{k}^{1}$ и $a_{l}^{2}$, где $k$ - количество параметров, представляющих деформацию отрыва, и $l-$ количество слагаемых, описывающих поперечный сдвиг. Для решения системы нелинейных уравнений необходимо задать начальные приближения для неизвестных коэффициентов $a_{k}^{1}$ и $a_{l}^{2}$. Если эти значения корректны, то функция $g_{m}$ обращается в нуль. В общем случае, $\begin{array}{lllll}\text { очевидно, что } & \text { функция } & g_{m} & \text { в }\end{array}$ не обратиться и следует прибегнуть к итерационной процедуре. Для реализации данной процедуры используется следующий подход. Неизвестные коэффициенты $a_{k}^{1}$ и $a_{l}^{2}$ находятся путем разложения функции $g_{m}$ в ряд Тейлора, в котором удерживаются слагаемые первого порядка относительно разностей.

Заменяя уравнение, описывающее поле напряжений вблизи вершины трещины, были получены неизвестные коэффициенты $a_{1}^{1}, a_{2}^{1}, a_{3}^{1}, \ldots ., a_{L}^{1}$ и $a_{1}^{2}, a_{2}^{2}, a_{3}^{2}, \ldots, a_{K}^{2}$, где $L$ - число, характеризующее количество слагаемых трещины типа I (трещины нормального отрыва); $K l-$ типа II (трещины поперечного сдвига). Чтобы решить систему нелинейных алгебраических уравнений, часто прибегают к переопределенному методу, суть которого заключается в линеаризации системы уравнений, следующей из оптико-механического закона (6). Линеаризация системы уравнений заключается в разложении полученной системы функций в окрестности нулевого (начального) приближения для коэффициентов $a_{1}^{1}, a_{2}^{1}, a_{3}^{1}, \ldots, a_{L}^{1}$ и $a_{1}^{2}, a_{2}^{2}, a_{3}^{2}, \ldots ., a_{K}^{2}$. Следующий этап - задание начального приближения коэффициентов, если эти приближения корректны, то функция $g_{m}$ обратится в нуль. 
Конечно, невозможно найти заранее правильные приближения для $a_{1}^{1}, a_{2}^{1}, a_{3}^{1}, \ldots ., a_{L}^{1}$ и $a_{1}^{2}, a_{2}^{2}, a_{3}^{2}, \ldots, a_{K}^{2}$, поэтому используется итерационный процесс для нахождения решения. В уравнении (7) приближение этих коэффициентов оценено посредством ряда Тейлора:

$$
\left(g_{m}\right)_{i+1}=\left(g_{m}\right)_{i}+\frac{\partial g_{m}}{\partial a_{1}^{1}} \Delta a_{1}^{1}+\frac{\partial g_{m}}{\partial a_{2}^{1}} \Delta a_{2}^{1}+\ldots+\frac{\partial g_{m}}{\partial a_{L}^{1}} \Delta a_{L}^{1}+\frac{\partial g_{m}}{\partial a_{1}^{2}} \Delta a_{1}^{2}+\frac{\partial g_{m}}{\partial a_{2}^{2}} \Delta a_{2}^{2}+\ldots+\frac{\partial g_{m}}{\partial a_{K}^{2}} \Delta a_{K}^{2},
$$

где нижний индекс $i$ - индекс итерации; $\Delta a_{1}^{1}, \Delta a_{2}^{1}, \Delta a_{3}^{1}, \ldots, \Delta a_{L}^{1}$ и $\Delta a_{1}^{2}, \Delta a_{2}^{2}, \Delta a_{3}^{2}, \ldots, \Delta a_{K}^{2} \quad-$ поправки для предыдущих оценок параметров $a_{1}^{1}, a_{2}^{1}, a_{3}^{1}, \ldots ., a_{L}^{1}$ и $a_{1}^{2}, a_{2}^{2}, a_{3}^{2}, \ldots ., a_{K}^{2}$.

Новые значения для масштабных множителей вычисляются путем решения уравнения $\left(g_{m}\right)_{i+1}=0$. Коэффициенты в последнем уравнении для каждой точки $m$ относительно коэффициентов $a_{1}^{1}, a_{2}^{1}, a_{3}^{1}, \ldots, a_{L}^{1}$ и $a_{1}^{2}, a_{2}^{2}, a_{3}^{2}, \ldots, a_{K}^{2}$ вычисляются согласно равенствам:

$$
\begin{aligned}
& \frac{\partial g_{m}}{\partial a_{n}^{1}}=\frac{1}{2}\left(\sigma_{11}-\sigma_{22}\right)_{m}\left(\frac{\partial \sigma_{11}}{\partial a_{n}^{1}}-\frac{\partial \sigma_{22}}{\partial a_{n}^{1}}\right)_{m}+2\left(\sigma_{12} \frac{\partial \sigma_{12}}{\partial a_{n}^{1}}\right)_{m}, \\
& \frac{\partial g_{m}}{\partial a_{n}^{2}}=\frac{1}{2}\left(\sigma_{11}-\sigma_{22}\right)_{m}\left(\frac{\partial \sigma_{11}}{\partial a_{n}^{2}}-\frac{\partial \sigma_{22}}{\partial a_{n}^{2}}\right)_{m}+2\left(\sigma_{12} \frac{\partial \sigma_{12}}{\partial a_{n}^{2}}\right)_{m} .
\end{aligned}
$$

Таким образом, необходимо получить решение системы уравнений $\{g\}_{i}=-[b]_{i}\{\Delta a\}_{i}$ и вектор поправок $\{\Delta a\}_{i}=-[c]_{i}^{-1}\{d\}_{i}$, где $[c]_{i}=[b]_{i}^{T}[b]_{i},\{d\}_{i}=[b]_{i}^{T}\{g\}_{i}$. На втором шаге итерации используется новая поправка $\{a\}_{i+1}=\{a\}_{i}+\{\Delta a\}_{i}$. Используя уравнения Алтури и Кобаяси относительно известных коэффициентов [7], получим уравнение:

$$
\begin{aligned}
& \left\{\begin{array}{l}
\frac{\partial \sigma_{11}}{\partial a_{n}^{1}} \\
\frac{\partial \sigma_{22}}{\partial a_{n}^{1}} \\
\frac{\partial \sigma_{12}}{\partial a_{n}^{1}}
\end{array}\right\}=\frac{n}{2} r^{(n-2) / 2}\left\{\begin{array}{l}
{\left[2+(-1)^{n}+\frac{n}{2}\right] \cos \left(\frac{n}{2}-1\right) \theta-\left(\frac{n}{2}-1\right) \cos \left(\frac{n}{2}-3\right) \theta} \\
\left.\left.2-(-1)^{n}-\frac{n}{2}\right] \cos \left(\frac{n}{2}-1\right) \theta+\left(\frac{n}{2}-1\right) \cos \left(\frac{n}{2}-3\right) \theta\right\} \\
-\left[(-1)^{n}+\frac{n}{2}\right] \sin \left(\frac{n}{2}-1\right) \theta+\left(\frac{n}{2}-1\right) \sin \left(\frac{n}{2}-3\right) \theta
\end{array}\right\} \\
& \left\{\begin{array}{l}
\frac{\partial \sigma_{11}}{\partial a_{n}^{2}} \\
\frac{\partial \sigma_{22}}{\partial a_{n}^{2}} \\
\frac{\partial \sigma_{12}}{\partial a_{n}^{2}}
\end{array}\right\}=\frac{n}{2} r^{(n-2) / 2}\left\{\begin{array}{l}
\left.\left.\left[\begin{array}{l}
\left.2+(-1)^{n}+\frac{n}{2}\right] \\
{\left[-(-1)^{n}-\frac{n}{2}\right.}
\end{array}\right] \cos \left(\frac{n}{2}-1\right) \theta-\left(\frac{n}{2}-1\right) \cos \left(\frac{n}{2}-3\right) \theta\right) \theta+\left(\frac{n}{2}-1\right) \cos \left(\frac{n}{2}-3\right) \theta\right\} \\
-\left[(-1)^{n}+\frac{n}{2}\right] \sin \left(\frac{n}{2}-1\right) \theta+\left(\frac{n}{2}-1\right) \sin \left(\frac{n}{2}-3\right) \theta
\end{array}\right\} .
\end{aligned}
$$

Производные от напряжений не зависят от неизвестных коэффициентов и не меняются с изменением шага итерации. Это неверно для производных от функции $g_{m}$, которые изменяются при каждой итерации. Первоначально векторы типа $\partial \sigma_{11} / \partial a_{n}^{1}$ вычисляются для каждых $\Delta a_{1}^{1}, \Delta a_{2}^{1}, \Delta a_{3}^{1}, \ldots, \Delta a_{L}^{1}$ и $\Delta a_{1}^{2}, \Delta a_{2}^{2}, \Delta a_{3}^{2}, \ldots, \Delta a_{K}^{2}$. Теперь можно построить 
матрицу $[b]$ и скорректировать значения $\{\Delta A\}_{i}$ и параметры, которые будут использоваться в следующей итерации.

В работе также выполнена серия экспериментов с помощью метода фотоупругости на пластинках с двумя коллинеарными трещинами одинаковой длины. Картина изохроматических полос изображена на рис. 2. На рис. 3 показана интерференционная картина в пластине с двумя коллинеарными наклонными трещинами для разных значений приложенной нагрузки. Выполнена цифровая обработка экспериментальной информации и найдены коэффициенты полного асимптотического разложения М. Уильямса. Точки, принадлежащие изохромам, найденные в ходе процедуры цифровой обработки данных и используемые для вычисления параметров механики разрушения, приведены на рис. 4 и отмечены зеленым цветом. Оказалось, что для экспериментального определения параметров механики разрушения следует прибегать к многопараметрическим асимптотическим разложениям механических полей. Результаты вычислений представлены в табл. 1-5. При удержании высших приближений значения коэффициентов интенсивности напряжений, соответствующие нормальному отрыву и поперечному сдвигу, $T$-напряжения быстро стабилизируются. Результаты аналитического решения хорошо согласуются с экспериментальными данными.
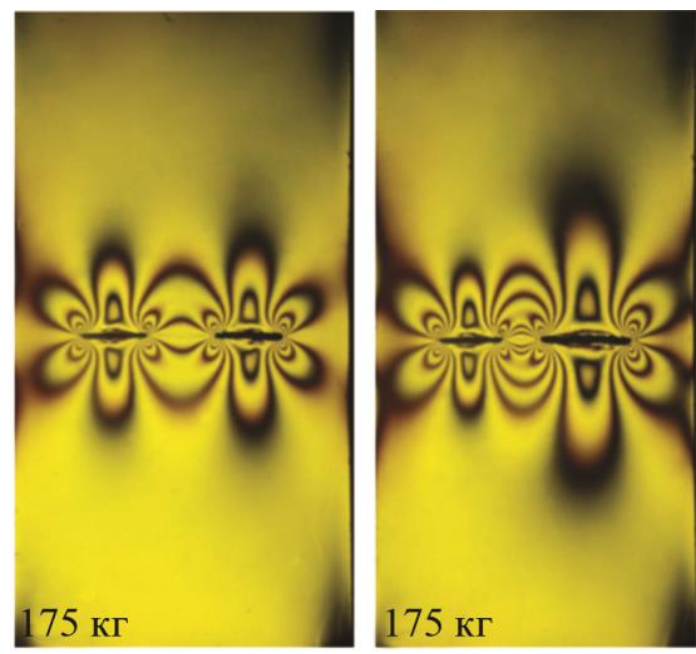

Рис. 2. Картины изохром в пластинах с двумя коллинеарными трещинами при разной интенсивности нагружения
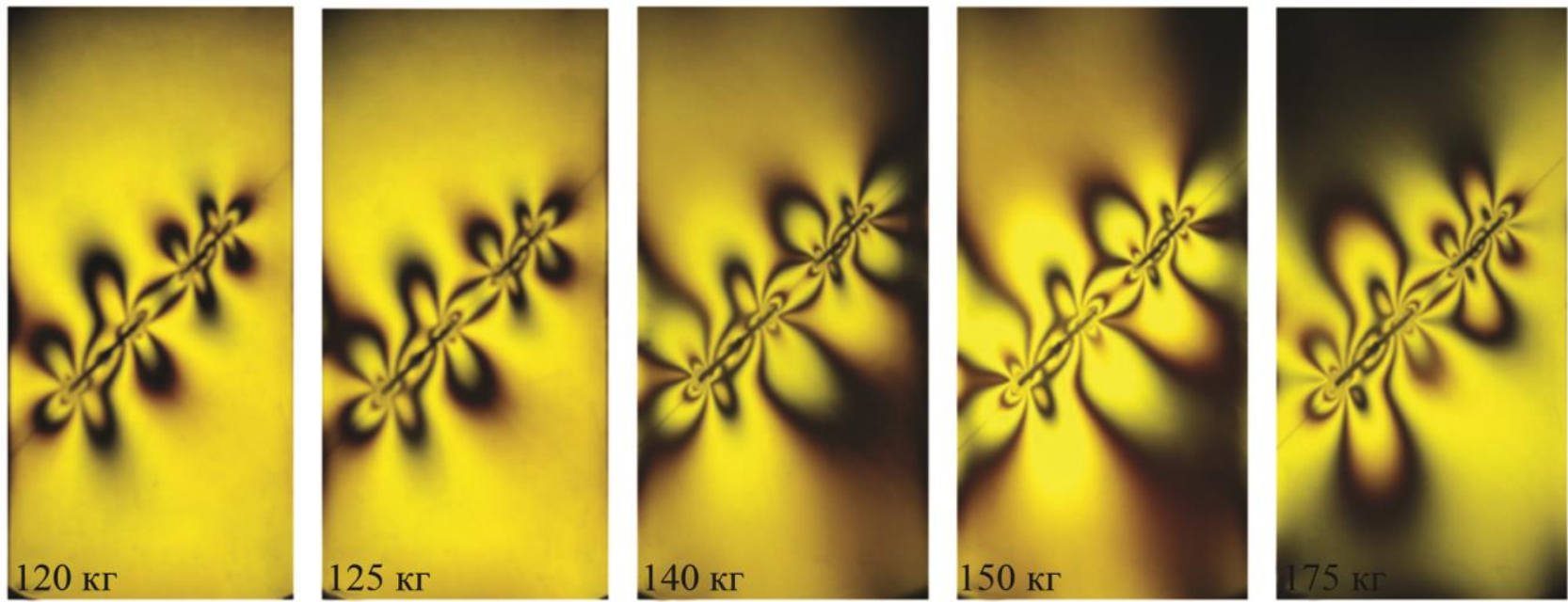

Рис. 3. Картины изохром в пластинах с двумя наклонными коллинеарными трещинами при разной интенсивности нагружения 


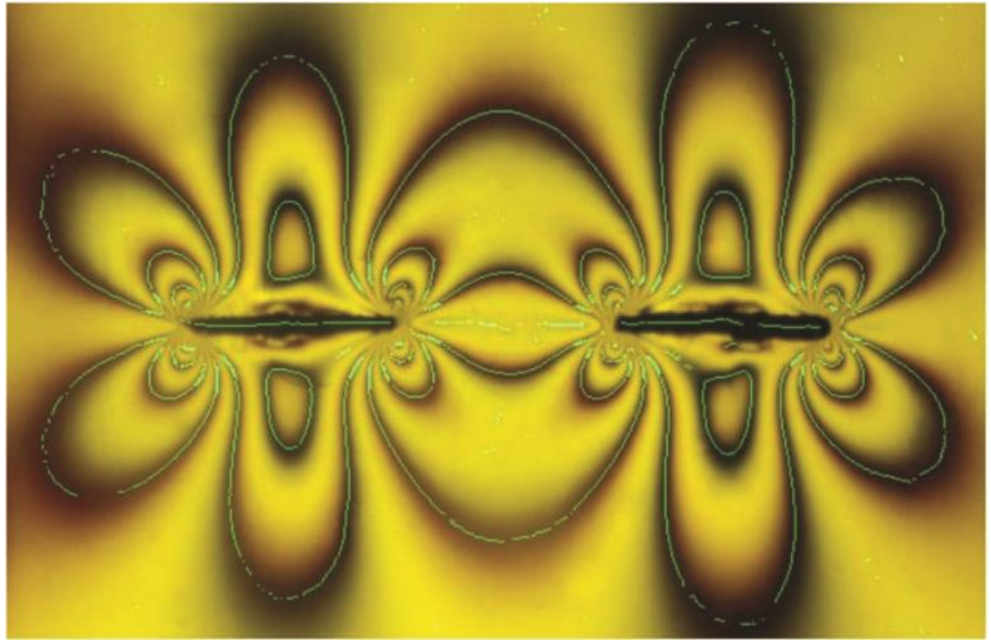

Рис. 4. Точки, принадлежащие изохромам, найденные в ходе процедуры цифровой обработки данных и используемые для вычисления параметров механики разрушения
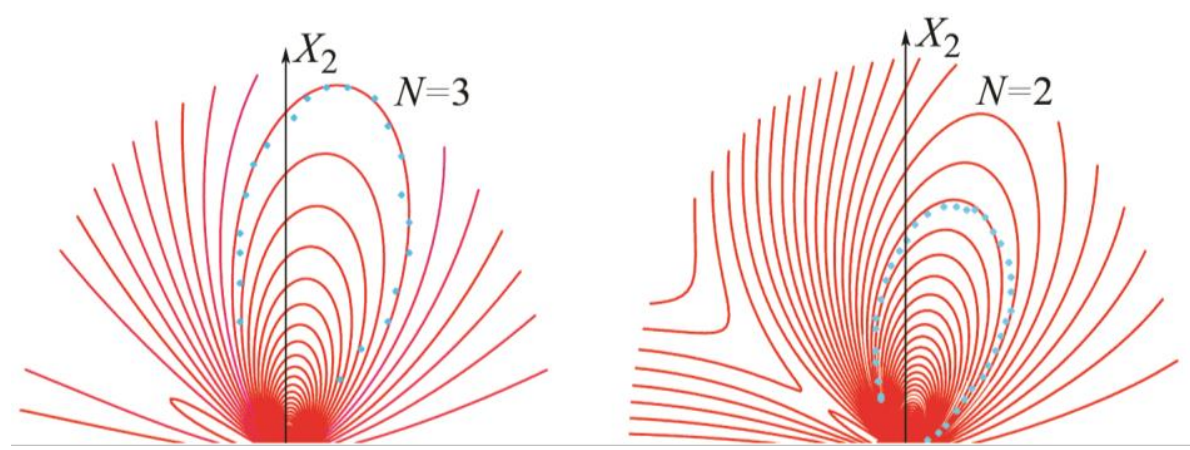

Рис. 5. Теоретически полученные изохроматические полосы (красный цвет) и экспериментальные точки (синий цвет)

На рис. 5 изображены теоретически полученные картины изохроматических полос для изохром различных порядков и экспериментальные точки.
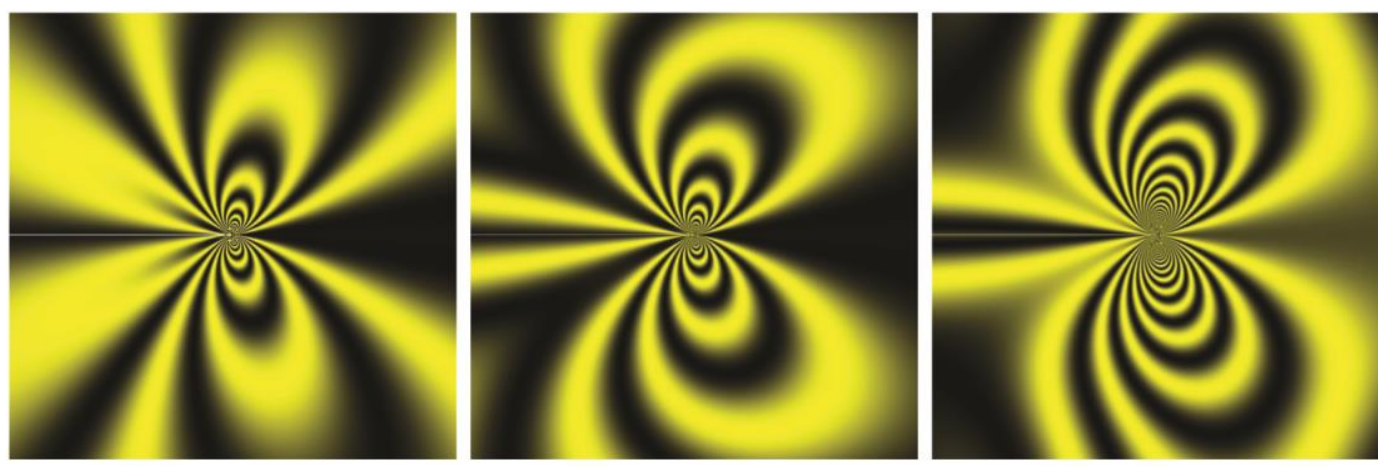

Рис. 6. Теоретически реконструированное поле изохроматических полос, полученное с помощью различного числа слагаемых в полном асимптотическом разложении М. Уильямса 
Таблица 1 - Коэффициенты полного асимтпотического разложения Уильямса у вершины трещины $z=a(a=1.5 \mathrm{~cm}, b=0.5 \mathrm{~cm}, c=-0.5 \mathrm{~cm}, d=-1.5 \mathrm{~cm})$

\begin{tabular}{|c|c|c|c|c|c|c|c|c|}
\hline$a_{1}^{1}\left(\mathrm{~cm}^{-3 / 2}\right)$ & $a_{2}^{1}\left(\mathrm{~cm}^{-2}\right)$ & $a_{3}^{1}\left(\mathrm{~cm}^{-5 / 2}\right)$ & $a_{5}^{1}\left(\mathrm{~cm}^{-7 / 2}\right)$ & $a_{7}^{1}\left(\mathrm{~cm}^{-9 / 2}\right)$ & $a_{9}^{1}\left(\mathrm{~cm}^{-11 / 2}\right)$ & $a_{11}^{1}\left(\mathrm{~cm}^{-13 / 2}\right)$ & $a_{13}^{1}\left(\mathrm{~cm}^{-15 / 2}\right)$ & $a_{15}^{1}\left(\mathrm{~cm}^{-17 / 2}\right)$ \\
\hline 0,5139 & $-0,2500$ & 0,2512 & $-0,0646$ & 0,0330 & $-0,0208$ & 0,0145 & $-0,0109$ & 0,0086 \\
\hline$a_{17}^{1}\left(\mathrm{~cm}^{-19 / 2}\right)$ & $a_{19}^{1}\left(\mathrm{~cm}^{-21 / 2}\right)$ & $a_{21}^{1}\left(\mathrm{~cm}^{-23 / 2}\right)$ & $a_{23}^{1}\left(\mathrm{~cm}^{-25 / 2}\right)$ & $a_{25}^{1}\left(\mathrm{~cm}^{-27 / 2}\right)$ & $a_{27}^{1}\left(\mathrm{~cm}^{-29 / 2}\right)$ & $a_{29}^{1}\left(\mathrm{~cm}^{-31 / 2}\right)$ & $a_{31}^{1}\left(\mathrm{~cm}^{-33 / 2}\right)$ & $a_{33}^{1}\left(\mathrm{~cm}^{-35 / 2}\right)$ \\
\hline$-0,0069$ & 0,0058 & $-0,0049$ & 0,00426 & $-0,0037$ & 0,0033 & $-0,0029$ & 0,0026 & $-0,0024$ \\
\hline$a_{35}^{1}\left(\mathrm{~cm}^{-37 / 2}\right)$ & $a_{37}^{1}\left(\mathrm{~cm}^{-39 / 2}\right)$ & $a_{39}^{1}\left(\mathrm{~cm}^{-41 / 2}\right)$ & $a_{41}^{1}\left(\mathrm{~cm}^{-43 / 2}\right)$ & $a_{43}^{1}\left(\mathrm{~cm}^{-45 / 2}\right)$ & $a_{45}^{1}\left(\mathrm{~cm}^{-47 / 2}\right)$ & $a_{47}^{1}\left(\mathrm{~cm}^{-49 / 2}\right)$ & $a_{49}^{1}\left(\mathrm{~cm}^{-51 / 2}\right)$ & $a_{51}^{1}\left(\mathrm{~cm}^{-53 / 2}\right)$ \\
\hline 0,0022 & $-0,0020$ & 0,0018 & $-0,0017$ & 0,0016 & $-0,00015$ & 0,0014 & $-0,0013$ & 0,0012 \\
\hline
\end{tabular}

Таблица 2 - Коэффициенты полного асимтпотического разложения Уильямса у вершины трещины $z=c \quad(a=1.5 \mathrm{~cm}, b=0.5 \mathrm{~cm}, c=-0.5 \mathrm{~cm}, d=-1.5 \mathrm{~cm})$

\begin{tabular}{|c|c|c|c|c|c|c|c|c|}
\hline$a_{1}^{1}\left(\mathrm{~cm}^{-3 / 2}\right)$ & $a_{2}^{1}\left(\mathrm{~cm}^{-2}\right)$ & $a_{3}^{1}\left(\mathrm{~cm}^{-5 / 2}\right)$ & $a_{5}^{1}\left(\mathrm{~cm}^{-7 / 2}\right)$ & $a_{7}^{1}\left(\mathrm{~cm}^{-9 / 2}\right)$ & $a_{9}^{1}\left(\mathrm{~cm}^{-11 / 2}\right)$ & $a_{11}^{1}\left(\mathrm{~cm}^{-13 / 2}\right)$ & $a_{13}^{1}\left(\mathrm{~cm}^{-15 / 2}\right)$ & $a_{15}^{1}\left(\mathrm{~cm}^{-17 / 2}\right)$ \\
\hline 0,52398 & $-0,25000$ & 0,27936 & $-0,04384$ & 0,04700 & $-0,00820$ & 0,00234 & $-0,00279$ & 0,001468 \\
\hline$a_{17}^{1}\left(\mathrm{~cm}^{-19 / 2}\right)$ & $a_{19}^{1}\left(\mathrm{~cm}^{-21 / 2}\right)$ & $a_{21}^{1}\left(\mathrm{~cm}^{-23 / 2}\right)$ & $a_{23}^{1}\left(\mathrm{~cm}^{-25 / 2}\right)$ & $a_{25}^{1}\left(\mathrm{~cm}^{-27 / 2}\right)$ & $a_{27}^{1}\left(\mathrm{~cm}^{-29 / 2}\right)$ & $a_{29}^{1}\left(\mathrm{~cm}^{-31 / 2}\right)$ & $a_{31}^{1}\left(\mathrm{~cm}^{-33 / 2}\right)$ & $a_{33}^{1}\left(\mathrm{~cm}^{-35 / 2}\right)$ \\
\hline$-0,00126$ & 0,01027 & $-0,00068$ & 0,00769 & $-0,00041$ & 0,00603 & $-0,00027$ & 0,00489 & $-0,00018$ \\
\hline$a_{35}^{1}\left(\mathrm{~cm}^{-37 / 2}\right)$ & $a_{37}^{1}\left(\mathrm{~cm}^{-39 / 2}\right)$ & $a_{39}^{1}\left(\mathrm{~cm}^{-41 / 2}\right)$ & $a_{41}^{1}\left(\mathrm{~cm}^{-43 / 2}\right)$ & $a_{43}^{1}\left(\mathrm{~cm}^{-45 / 2}\right)$ & $a_{45}^{1}\left(\mathrm{~cm}^{-47 / 2}\right)$ & $a_{47}^{1}\left(\mathrm{~cm}^{-49 / 2}\right)$ & $a_{49}^{1}\left(\mathrm{~cm}^{-51 / 2}\right)$ & $a_{51}^{1}\left(\mathrm{~cm}^{-53 / 2}\right)$ \\
\hline 0,00407 & $-0,00013$ & 0,00345 & $-0,00009$ & 0,00298 & $-0,00007$ & 0,00260 & $-0,00005$ & 0,00230 \\
\hline
\end{tabular}

Таблица 3 - Коэффициенты полного асимтпотического разложения Уильямса у вершины трещины $z=a(a=2 \mathrm{~cm}, b=0.5 \mathrm{~cm}, c=-0.5 \mathrm{~cm}, d=-1.5 \mathrm{~cm})$

\begin{tabular}{|c|c|c|c|c|c|c|c|c|}
\hline$a_{1}^{1}\left(\mathrm{~cm}^{-3 / 2}\right)$ & $a_{2}^{1}\left(\mathrm{~cm}^{-2}\right)$ & $a_{3}^{1}\left(\mathrm{~cm}^{-5 / 2}\right)$ & $a_{5}^{1}\left(\mathrm{~cm}^{-7 / 2}\right)$ & $a_{7}^{1}\left(\mathrm{~cm}^{-9 / 2}\right)$ & $a_{9}^{1}\left(\mathrm{~cm}^{-11 / 2}\right)$ & $a_{11}^{1}\left(\mathrm{~cm}^{-13 / 2}\right)$ & $a_{13}^{1}\left(\mathrm{~cm}^{-15 / 2}\right)$ & $a_{15}^{1}\left(\mathrm{~cm}^{-17 / 2}\right)$ \\
\hline 0,62576 & $-0,25000$ & 0,20400 & $-0,03470$ & 0,01181 & $-0,00499$ & 0,00234 & $-0,00117$ & 0,00061 \\
\hline$a_{17}^{1}\left(\mathrm{~cm}^{-19 / 2}\right)$ & $a_{19}^{1}\left(\mathrm{~cm}^{-21 / 2}\right)$ & $a_{21}^{1}\left(\mathrm{~cm}^{-23 / 2}\right)$ & $a_{23}^{1}\left(\mathrm{~cm}^{-25 / 2}\right)$ & $a_{25}^{1}\left(\mathrm{~cm}^{-27 / 2}\right)$ & $a_{27}^{1}\left(\mathrm{~cm}^{-29 / 2}\right)$ & $a_{29}^{1}\left(\mathrm{~cm}^{-31 / 2}\right)$ & $a_{31}^{1}\left(\mathrm{~cm}^{-33 / 2}\right)$ & $a_{33}^{1}\left(\mathrm{~cm}^{-35 / 2}\right)$ \\
\hline$-0,00033$ & 0,00018 & $-0,00010$ & 0,00005 & $-0,00003$ & 0,00002 & $-0,00001$ & $0.733 \cdot 10^{-5}$ & $-0.442 \cdot 10^{-5}$ \\
\hline
\end{tabular}


Таблица 4 - Коэффициенты полного асимтпотического разложения Уильямса у вершины трещины $z=d \quad(a=1.5 \mathrm{~cm}, b=0.5 \mathrm{~cm}, c=-0.5 \mathrm{~cm}, d=-1.5 \mathrm{~cm})$

\begin{tabular}{|c|c|c|c|c|c|c|c|c|}
\hline$a_{1}^{1}\left(\mathrm{~cm}^{-3 / 2}\right)$ & $a_{2}^{1}\left(\mathrm{~cm}^{-2}\right)$ & $a_{3}^{1}\left(\mathrm{~cm}^{-5 / 2}\right)$ & $a_{5}^{1}\left(\mathrm{~cm}^{-7 / 2}\right)$ & $a_{7}^{1}\left(\mathrm{~cm}^{-9 / 2}\right)$ & $a_{9}^{1}\left(\mathrm{~cm}^{-11 / 2}\right)$ & $a_{11}^{1}\left(\mathrm{~cm}^{-13 / 2}\right)$ & $a_{13}^{1}\left(\mathrm{~cm}^{-15 / 2}\right)$ & $a_{15}^{1}\left(\mathrm{~cm}^{-17 / 2}\right)$ \\
\hline 0,52397 & $-0,25000$ & 0,27937 & $-0,04384$ & 0,04700 & $-0,00820$ & 0,02343 & $-0,00279$ & 0,014687 \\
\hline$a_{17}^{1}\left(\mathrm{~cm}^{-19 / 2}\right)$ & $a_{19}^{1}\left(\mathrm{~cm}^{-21 / 2}\right)$ & $a_{21}^{1}\left(\mathrm{~cm}^{-23 / 2}\right)$ & $a_{23}^{1}\left(\mathrm{~cm}^{-25 / 2}\right)$ & $a_{25}^{1}\left(\mathrm{~cm}^{-27 / 2}\right)$ & $a_{27}^{1}\left(\mathrm{~cm}^{-29 / 2}\right)$ & $a_{29}^{1}\left(\mathrm{~cm}^{-31 / 2}\right)$ & $a_{31}^{1}\left(\mathrm{~cm}^{-33 / 2}\right)$ & $a_{33}^{1}\left(\mathrm{~cm}^{-35 / 2}\right)$ \\
\hline$-0,00126$ & 0,01027 & $-0,000068$ & 0,00769 & $-0,00041$ & 0,00603 & $-0,00026$ & 0,00489 & $-0,00018$ \\
\hline$a_{35}^{1}\left(\mathrm{~cm}^{-37 / 2}\right)$ & $a_{37}^{1}\left(\mathrm{~cm}^{-39 / 2}\right)$ & $a_{39}^{1}\left(\mathrm{~cm}^{-41 / 2}\right)$ & $a_{41}^{1}\left(\mathrm{~cm}^{-43 / 2}\right)$ & $a_{43}^{1}\left(\mathrm{~cm}^{-45 / 2}\right)$ & $a_{45}^{1}\left(\mathrm{~cm}^{-47 / 2}\right)$ & $a_{47}^{1}\left(\mathrm{~cm}^{-49 / 2}\right)$ & $a_{49}^{1}\left(\mathrm{~cm}^{-51 / 2}\right)$ & $a_{51}^{1}\left(\mathrm{~cm}^{-53 / 2}\right)$ \\
\hline 0,00407 & $-0,00013$ & 0,00345 & $-0,00009$ & 0,00298 & $-0,00007$ & 0,00260 & $-0,00005$ & 0,00230 \\
\hline
\end{tabular}

Таблица 5 - Коэффициенты полного асимтпотического разложения Уильямса у вершины трещины $z=d \quad(a=2.5 \mathrm{~cm}, b=0.5 \mathrm{~cm}, c=-0.5 \mathrm{~cm}, d=-1.5 \mathrm{~cm})$

\begin{tabular}{|c|c|c|c|c|c|c|c|c|}
\hline$a_{1}^{1}\left(\mathrm{~cm}^{-3 / 2}\right)$ & $a_{2}^{1}\left(\mathrm{~cm}^{-2}\right)$ & $a_{3}^{1}\left(\mathrm{~cm}^{-5 / 2}\right)$ & $a_{5}^{1}\left(\mathrm{~cm}^{-7 / 2}\right)$ & $a_{7}^{1}\left(\mathrm{~cm}^{-9 / 2}\right)$ & $a_{9}^{1}\left(\mathrm{~cm}^{-11 / 2}\right)$ & $a_{11}^{1}\left(\mathrm{~cm}^{-13 / 2}\right)$ & $a_{13}^{1}\left(\mathrm{~cm}^{-15 / 2}\right)$ & $a_{15}^{1}\left(\mathrm{~cm}^{-17 / 2}\right)$ \\
\hline 0,56275 & $-0,25000$ & 0,31993 & $-0,02563$ & 0,06089 & $-0,00065$ & 0,02999 & 0,00117 & 0,01853 \\
\hline$a_{17}^{1}\left(\mathrm{~cm}^{-19 / 2}\right)$ & $a_{19}^{1}\left(\mathrm{~cm}^{-21 / 2}\right)$ & $a_{21}^{1}\left(\mathrm{~cm}^{-23 / 2}\right)$ & $a_{23}^{1}\left(\mathrm{~cm}^{-25 / 2}\right)$ & $a_{25}^{1}\left(\mathrm{~cm}^{-27 / 2}\right)$ & $a_{27}^{1}\left(\mathrm{~cm}^{-29 / 2}\right)$ & $a_{29}^{1}\left(\mathrm{~cm}^{-31 / 2}\right)$ & $a_{31}^{1}\left(\mathrm{~cm}^{-33 / 2}\right)$ & $a_{33}^{1}\left(\mathrm{~cm}^{-35 / 2}\right)$ \\
\hline 0,00122 & 0,01286 & 0,00106 & 0,00958 & 0,00901 & 0,00749 & 0,00766 & 0,00060 & 0,00065 \\
\hline$a_{35}^{1}\left(\mathrm{~cm}^{-37 / 2}\right)$ & $a_{37}^{1}\left(\mathrm{~cm}^{-39 / 2}\right)$ & $a_{39}^{1}\left(\mathrm{~cm}^{-41 / 2}\right)$ & $a_{41}^{1}\left(\mathrm{~cm}^{-43 / 2}\right)$ & $a_{43}^{1}\left(\mathrm{~cm}^{-45 / 2}\right)$ & $a_{45}^{1}\left(\mathrm{~cm}^{-47 / 2}\right)$ & $a_{47}^{1}\left(\mathrm{~cm}^{-49 / 2}\right)$ & $a_{49}^{1}\left(\mathrm{~cm}^{-51 / 2}\right)$ & $a_{51}^{1}\left(\mathrm{~cm}^{-53 / 2}\right)$ \\
\hline 0,00504 & 0,00057 & 0,00027 & 0,00050 & 0,00368 & 0,00044 & 0,00321 & 0,00039 & 0,00284 \\
\hline
\end{tabular}


На рис. 6 показано теоретически реконструированное поле изохроматических полос, полученное с помощью различного числа слагаемых в полном асимптотическом разложении М. Уильямса. На рис. 6 слева показаны изохроматические полосы, полученные с помощью двучленного асимптотического разложения М. Уильямса, в центре картина изохроматических полос построена с помощью пятичленного асимптотического разложения М. Уильямса. Справа изображение получено посредством девятичленного асимптотического разложения поля напряжений у вершины трещины.

\section{Выводы и обсуждение результатов}

Работа посвящена аналитическому определению коэффициентов полного асимптотического разложения М. Уильямса у вершин двух коллинеарных трещин в бесконечной пластине, находящейся в условиях смешанного нагружения. Представлен метод вычисления коэффициентов асимптотического разложения М. Уильямса, базирующийся на классическом комплексном представлении решения Колосова-Мусхелишвили. Метод позволяет получить аналитические выражения для коэффициентов интенсивности напряжений, $T$-напряжений и коэффициентов высших приближений в разложении М. Уильямса. Найдены аналитические зависимости коэффициентов полного асимптотического разложения М. Уильямса от приложенной нагрузки, длин трещин и расстояний между ними для пластины с двумя коллинеарными трещинами. С помощью метода фотоупругости выполнено экспериментальное исследование пластин с двумя коллинеарными трещинами. Проведена цифровая обработка экспериментальной информации, с помощью которой вычислены коэффициенты полного асимптотического разложения М. Уильямса у вершин трещин. Проведена серия конечноэлементных расчетов в CAE пакете SIMULIA Abaqus для численного определения коэффициентов асимптотического разложения М.Уильямса. МКЭ расчеты подтверждают полученные аналитические выражения.

\section{Литература}

1. Hello G., Tahar M.-B., Roelandt J.-M. Analytical determination of coefficients in crack-tip stress expansions for a finite crack in an infinite plane medium // International Journal of Solids and Structures. - 2012. - Vol. 49, iss. 3-4. - P. 556-566. - DOI: 10.1016/j.ijsolstr.2011.10.024.

2. Степанова Л. В. Математические методы механики разрушения. - Самара : Самарский университет, 2006. - 232 с.

3. Мусхелишвили Н. И. Некоторые основные задачи математической теории упругости. М. : Наука, 1966. - 708 с.

4. Gupta M., Alderliesten R. C., Benedictus R. A review of T-stress and its effects in fracture mechanics // Engineering Fracture Mechanics. - 2015. - Vol. 134. - P. 218-241. DOI: 10.1016/j.engfracmech.2014.10.013.

5. Matvienko $\mathrm{Yu}$. G. The effect of the non-singular t-stress components on crack tip plastic zone under mode I loading // Procedia Materials Science. - 2014. - Vol. 134. - P. 141-146. DOI: 10.1016/j.mspro.2014.06.026.

6. Stepanova L. V., Adylina E. M. Stress-strain state in the vicinity of a crack tip under mixed loading // Journal of Applied Mechanics and Technical. - 2014. - Vol. 55, iss. 5. - P. 181-194. DOI: 10.1134/S0021894414050186.

7. Экспериментальная механика : в 2-х книгах / пер. с англ. под ред. А. Кобаяси. - М. : Мир, 1990. 616 с. - Кн. 1. 